\title{
Conf-931048-9
}

Revised 1/20/94

UCRL-JC-114969

PREPRINT

\section{A Comparison of Driver Concepts for Heavy-Ion Fusion}

\author{
W. M. Sharp \\ Lawrence Livermore National Laboratory, University of California \\ P.O. Box 5508, L-440, Livermore Califormia 94550, USA
}

This paper was prepared for submittal to the

Proceedings of the 11th Workshop on Laser Interaction and Related Plasma Phenomena, Monterey, CA, October 25-29, 1993

January 10, 1994

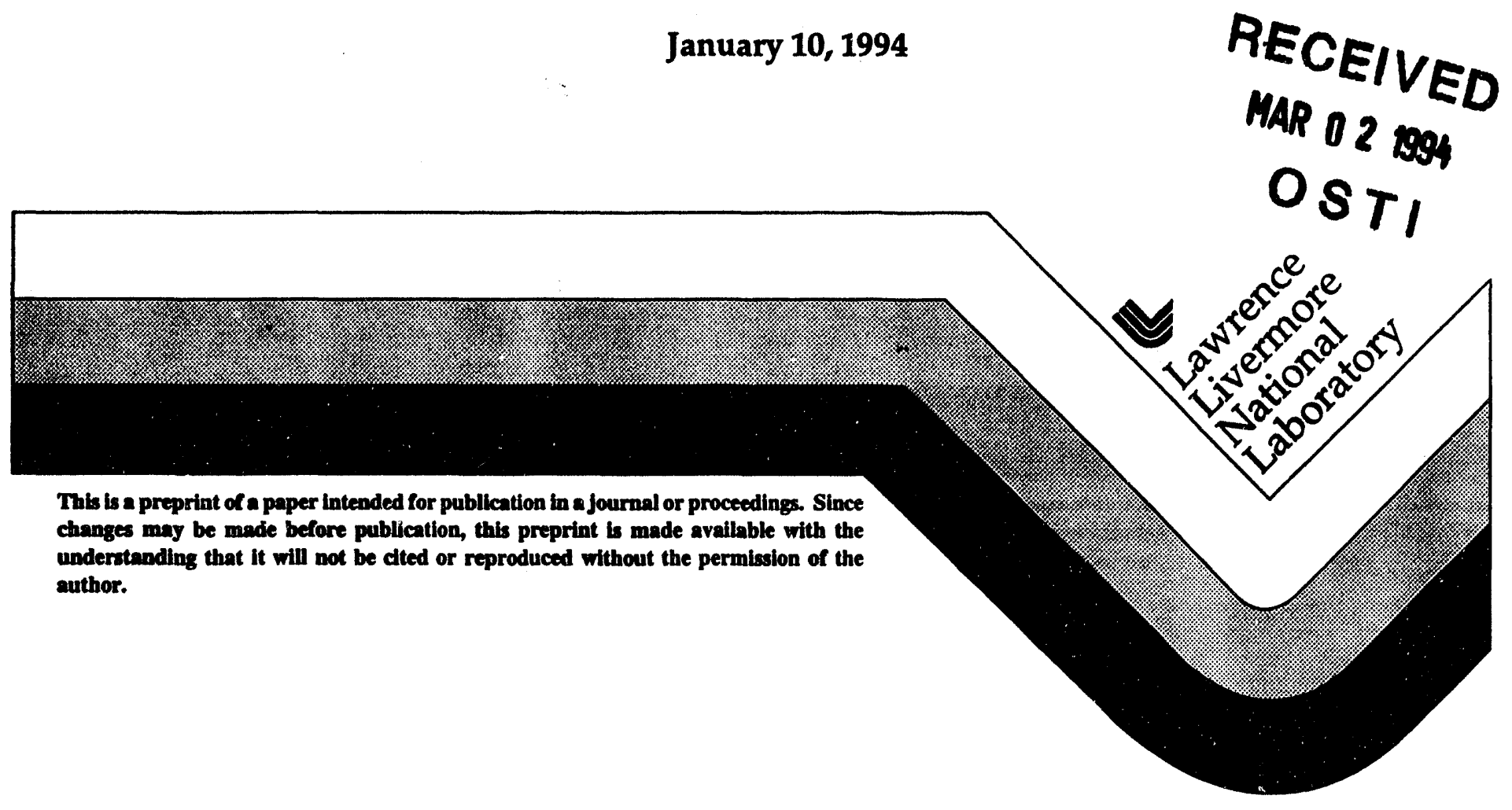

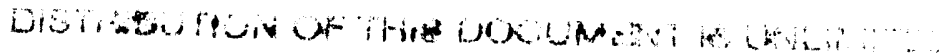




\section{DISCLAIMER}

This document was prepared as an acoount of work sponsored by an agency of the United Strtes Govemment. Neither the United Stules Government nor the University of Califomin nor eny of their employees, makes any warrenty, express or implied, or assumes any legal liability or responsibility for the accuracy. complesenets, or usefulness of any information, spparatus, product, or process dieclosed, or represents thet its use would not infringe privately owned rights. Reference herein to any specific commercial products, process, or service by trade name, trademark, manufacturer, or otherwise, does not necessarily conatitute or imply its endorsement, recommendation, or favoring by the United States Govemment or the University of Califomia. The views and opinions of authors expressed herein do nol necessarily state or reflect those of the Uniced States Govemment or the University of Califomia, and shall not be used for advertising or product endorsement purposes. 


\title{
A COMPARISON OF DRIVER CONCEPTS FOR HEAVY-ION FUSION ${ }^{\dagger}$
}

\author{
W. M. Sharp \\ Lawrence Livermore National Laboratory, Livermore, CA 94550
}

\begin{abstract}
The indirect-drive targets being considered for inertial fusion require the driver to deposit around $5 \mathrm{MJ}$ on a target in less than 10 ns. This requirement can in principle be met by heavy-ion beams with particle masses between 120 and 240 amu, an ion kinetic energy in the range of 6-12 GeV, and a total current in excess of $30 \mathrm{kA}$. Three strategies for generating beams with these parameters are currently being studied. European laboratories are investigating the use of low-current beams from a radio-frequency accelerator. To obtain the needed current density, these beams would be stacked and accumulated in storage rings and then directed simultaneoully at the target. American researchers are developing high-current induction accelerators, and the two principal configurations under consideration are the linear driver and the "recirculator," in which ion pulses pass repeatedly through the same accelerator elements. The merits of the three approaches are compared, and key physics uncertainties in each are identified.
\end{abstract}

\section{INTRODUCTION}

The basic concern of inertial fusion is depositing enough energy in the shell of a target in a sufficiently short time. Heavy-ion accelerators are an attractive candidate for a driver for inertial-fusion energy, both because their beams can transfer energy efficiently to targets, and because they can deliver large peak powers with manageable currents.

One problem confronting ion drivers is the scaling of target gain, usually defined as the ratio of fusion energy generated to the input kinetic energy. Gain typically increases with increasing energy on target, decreasing beam focal-spot size, and decreasing range, which corresponds to a lower energy per ion. In contrast, ion accelerators are conventionally designed to deliver low current and high energy per ion, and, at higher currents, the beam space charge and transverse temperature tend to give a large spot size. The engineering problem for heavy-ion drivers is to obtain sufficiently concentrated energy while still focusing, steering, and finally aiming the beam. The economic problem is doing this at a cost that is competitive with other energy sources.

In this paper, the principal concepts for heavy-ion drivers are presented and briefly compared. Target requirements are used in Section 2 to choose a typical set of accelerator specifications, and the following two sections review the driver approaches being taken by European and American research groupsi. Experimental work on heavy-ion fusion (HIF) is discussed in a final section.

\section{DRIVER REQUIREMENTS}

\subsection{Nominal Accelerator Parameters}

Many requirements for heavy-ion drivers are set by the target physics. The target type currently favored by most researchers is an "indirect-drive" configuration, sketched in Fig. 1a, in which the fuel pellet is centered in a shell or "hohlraum" made of a material with a high atomic number $Z$. The beams are focused so as to heat one or more "absorbers" or "converters," which then radiate soft X-rays into the hohlraum. The X-ray flux is nearly isotropic due to the large hohlraum wall area and the multiple reflections inside the high $Z$ shell, and the radiation pressure compresses and heats the pellet. In typical designs, the pellet is a hollow shell of solid Deuterium-Tritium (DT) mixture surrounded by an "ablator," a layer of low- $Z$ material such as Beryllium or plastic. As the ablator heats, the outer layer blows off, and the reaction force isobarically

$\uparrow$ This work was performed under the auspices of the US Department of Energy by Lawrence Livermore National Laboratory under W-7405-ENG-48. 


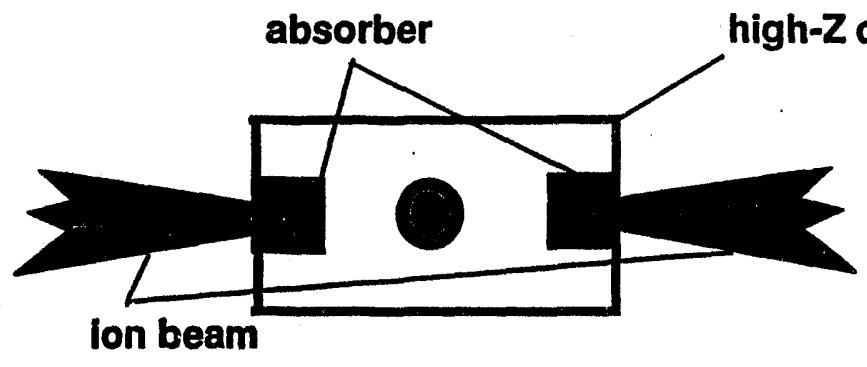

(a)

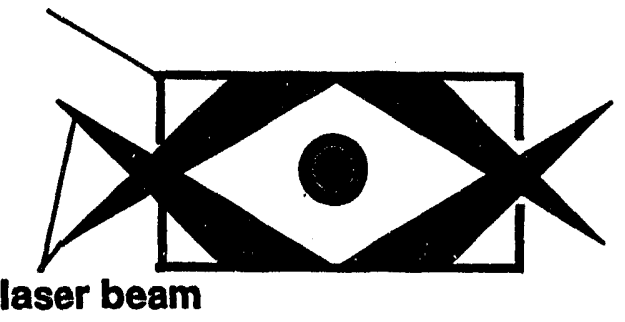

(b)

Fig. 1 Schematic layout of inertial-fusion targets: (a) heavy-ion target and (b) laser target

pushes the DT toward the center. A "hot spot" develops inside the compressing shock front and finally ignites the fuel. This target design is very similar to a type used with laser drivers, shown schematically in Fig. 1b.

The behavior of indirect-drive targets is complicated and depends on such parameters as the hohlraum geometry and material, the details of pellet design, and the beam energy and pulse shape. Nonetheless, many beam parameters can be inferred from relatively simple considerations. For a given ion, the absorber thickness and material determine the required kinetic energy per ion, and the absorber surface area directly sets the beam spot size. The pulse duration and required energy deposition can be estimated from the pellet size and the implosion velocity. Simple hydrodynamics arguments ${ }^{(1)}$ suggest that isobaric compression can be achieved with an implosion velocity $V_{i m p}$ of about $3 \times 10^{7} \mathrm{~cm} / \mathrm{s}, 80$ if the inner radius of the fuel pellet is $3 \mathrm{~mm}$, the pulse duration is about $10 \mathrm{~ns}$. Likewise, for a fuel mass $M_{f}$ of $7 \mathrm{mg}$, the implosion energy $\frac{1}{2} M, V_{i m p}^{2}$ is roughly $6 \times 10^{5} \mathrm{~J}$. Energy is lost in converting the beam energy to X-rays, in ablating the outer layer of the pellet, and in hydrodynamic drag, and the implosion efficiency is the fraction of the input beam energy that remains as kinetic energy of the fuel. If this efficiency is estimated to be about $15 \%$, then the required beam energy on target is about $4 \mathrm{MJ}$, and the corresponding average power on target is $4 \times 10^{14} \mathrm{~W}$, about $\mathbf{4 0}$ times the average worldwide electric power consumption.

From these simple target considerations, we take the nominal beam parameters to be those in Table 1 . These parameters are in no sense optimized, and they clearly depend on the target design. If a target with higher gain or better energy coupling could be devised, for example, the energy required on target could be reduced. The choice of an ion mass of about $200 \mathrm{amu}$ is largely arbitrary. Lower mass ions could equally be used, but because stopping power in an absorber is proportional to the inverse square of the ion velocity, the energy per ion must be lower with lighter ions, necessitating a higher particle current to obtain the needed power on target. The required current increases inversely with decreasing ion mass, and for ions lighter than about 120 amu, ballistic transport to the target becomes impractical due to the buildup of space-charge near the target. Finally, the $3 \mathrm{~mm}$ focal-spot radius is a compromise between two conflicting demands. A larger spot size would be easier to hit but would reduce target gain because of the greater absorber volume to be heated. However, a significantly smaller spot size would require that the four-dimensional transverse phase-space volume of the beam, usually characterized by an "emittance" in two orthogonal directions, be unrealistically small.

Table 1 Nominal parameters for a HIF driver on target

$\begin{array}{ll}\text { ion mass } & 200 \mathrm{amu} \\ \text { pulse energy } & 4 \mathrm{MJ} \\ \text { pulse duration } & 10 \mathrm{~ns} \\ \text { focal-spot radius } & 3 \mathrm{~mm} \\ \text { kinetic energy/ion } & 10 \mathrm{GeV} \\ \text { total current } & 40 \mathrm{kA} \\ \text { total power } & 4 \times 10^{14} \mathrm{~W}\end{array}$




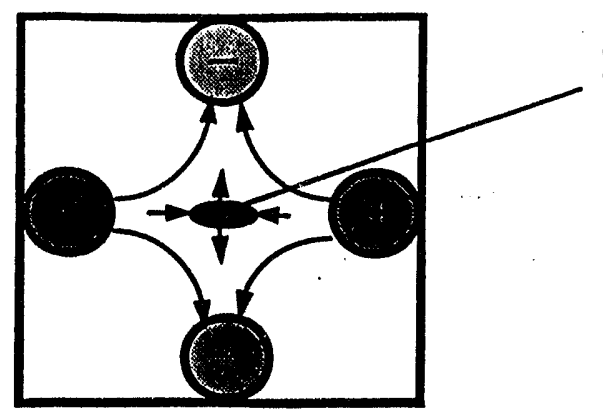

(a)

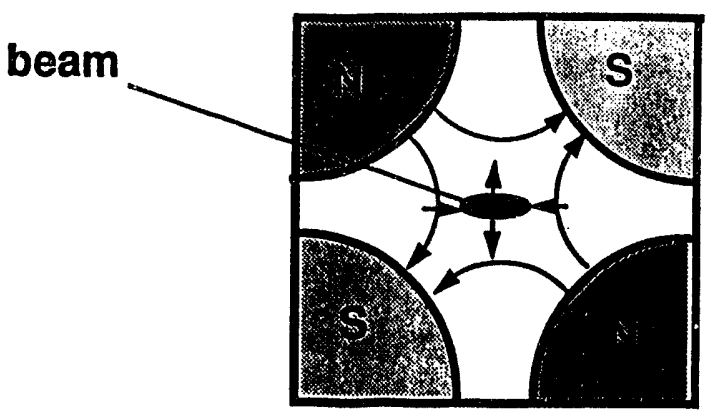

(b)

Fig. 2 Schematic layout of (a) electrostatic and (b) magnetic quadrupoles

\subsection{Driver Concepts}

Two fundamentally different HIF driver concepts have been advanced to satisfy the requirements for inertial fusion discussed above. European and Japanese work has focused on radio-frequency (rf) accelerators. These accelerators can easily produce ions of the required energy, but due to their low current, the beams must first be accumulated and then compressed in time to give the required power on target. In contrast, American conceptual designs for heavy-ion drivers use induction accelerators, which produce much higher currents than if accelerators but operate with lower accelerating gradients. The two principal configurations suggested to date for induction drivers are a linear layout, in which the beams pass through each accelerator element once, and a recirculating geometry, where beams are sent repeatedly through the same accelerator elements.

Although the two types of heavy-ion accelerator are quite different, both propose to use quadrupole or "alternating-gradient" transverse focusing. Focusing is needed in the transverse plane to balance the cutward force due to the beam space charge and transverse emittance. A quadrupole focusing element squeezes the beam in one of the directions in the transverse plane, while it defocuses the beam in the orthogonal direction. If the focusing and defocusing forces increase with distance from the accelerator axis, a periodic series of quadrupoles, focusing alternately in the two directions, gives a net focusing force because the beam is larger along the focusing axis. The two most commonly used types of quadrupole focusing are electrostatic, sketched in Fig. 2a, and magnetic, shown in Fig. 2b. Since the magnetic force is proportional to the velocity of ions, magnetic quadrupoles are invariably used at higher energies, but electrostatic quadrupoles become economically favorable below beam velocities of about $V_{b} / c \sim 0.02$. Nonetheless, either type of focusing could be used over a broad range of velocities around this value. A conservative upper bound on the current that can be transported is found by equating the space-charge force at the beam edge with the quadrupole strength there, averaged over a focusing period. For magnetic quadrupoles with a pole-tip strength of $B_{q}$ and an aperture of $R$, this so-called "Maschke limit" can be written ${ }^{(2)}$ in SI units

$$
I_{\max } \approx \frac{\pi^{2} \epsilon_{0}}{4} a^{2} V_{b}^{2} \frac{B_{q}}{R}
$$

where $q$ is the beam charge state and $a$ is the average beam radius. An analogous expression for electrostatic quadrupoles is obtained by substituting $E_{q} / V_{b}$ for $B_{q}$, where $E_{q}$ is the pole-tip electric field. Since $B_{q}$ can exceed $5 \mathrm{~T}$ for superconducting quadrupoles, the maximum current from Eq. (1) is about $10 \mathrm{kA}$, so four or more beams are required to deliver the required $4 \times 10^{14} \mathrm{~W}$ power on target at $10 \mathrm{GeV}$.

\section{RADIO-FREQUENCY DRIVERS}

\subsection{RF Accelerators}

In $r$ ion accelerators, particles are accelerated by the axial electric field of a radio-frequency wave in a standing-wave cavity. A high-power rf signal at the chosen frequency is fed into a cavity, called an "Alvarez 


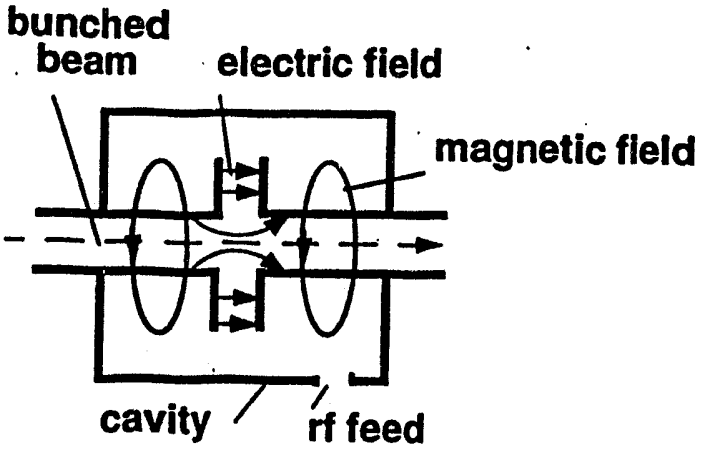

(a)

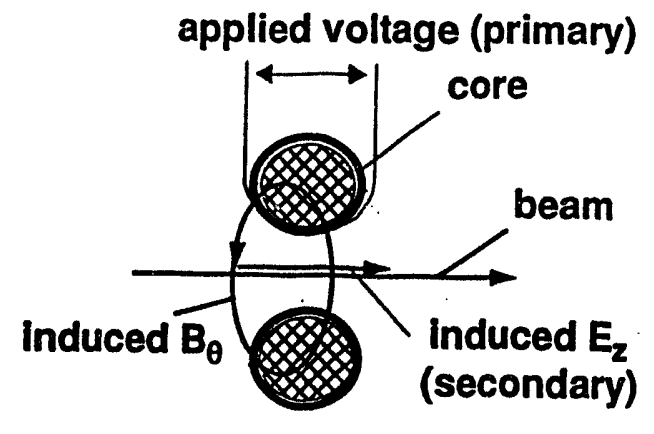

(b)

Fig. 3 Schematic layouts of (a) a cavity of a standing-wave radio-frequency accelerator and (b) a cell of an induction accelerator

structure,"(3) such as the one sketched in Fig. 3a. The wave magnetic field is azimuthal, and the axial component of the electric field concentrates in one or, more often, several gaps in a coaxial beam pipe. The electric field in a gap varies sinusoidally at the signal frequency, which can range typically from about $20 \mathrm{MHz}$ to a few GHz. In order that ions in the gaps are always accelerated, they must be bunched into "micropulses" that are shorter than half an $\mathrm{rf}$ wavelength and separated by an integral multiple of the gap spacing. The gap spacing, likewise, should equal the distance the beam drifts in some multiple of the wave period. To maintain correct phasing as an ion beam accelerates, the gap spacing must be chosen appropriately for each gap. One useful feature of an if accelerator is that it can provide longitudinal focusing of micropulses. If a pulse is timed to coincide with the rising portion of the sinusoidal electric field, then ions in the front of a micropulse are accelerated less than mid-pulse ions, while ions near the end are accelerated more. The rf field, in effect, forms a ponderomotive potential well or "bucket" that is a quarter wave length long, and a properly timed micropulse remains trapped in this bucket while gaining energy.

Two features of if ion accelerators strongly affect their application as heavy-ion drivers. The first is that such accelerators are normally constant-current devices. Since charge in a micropulse remains constant during acceleration and the time separation between micropulses is some constant multiple of the wave period, the average current also remains constant during acceleration, and the beam cannot be compressed as it is accelerated. The second pertinent feature of $\mathrm{rf}$ accelerators is that the maximum average current is typically limited by economics to about $100 \mathrm{~mA}$. At higher currents space charge results in a larger beam radius and correspondingly larger standing-wave structures, driving up the accelerator cost. Also, wider gaps are needed with a larger beam pipe to allow the electric field to penetrate to the axis, so a lower frequency must be used to allow micropulses to cross the gaps at the correct if phase. The reduced acceleration gradient resulting from the lower frequency increases the accelerator length and cost. Because the current provided by an rf accelerator is much less than the required driver current on target, the current must either be collected simultaneously from several accelerators or the current from one accelerator must be compressed in time by a factor of at least $4 \times 10^{5}$.

\subsection{RF Driver Layout}

Current European designs for HIF accelerators specify a single rf accelerator in combination with storage rings to obtain the required total cur:ent. This approach has several advantages. RF accelerator technology has been used in high-energy accelerators for about forty years, and obtaining the required energy per ion is straightforward due to the relatively high accelerating gradients $(\sim 10 \mathrm{MeV} / \mathrm{m})$ in rf accelerators. Also, the approach avoids the manipulation of beams with high space charge, which is a potential source of emittance increase.

The major problem designing an rf driver with storage-ring accumulation is obtaining the required time compression. If the output from a single accelerator is collected before compression, than an accumulation time of at least $4 \mathrm{~ms}$ is needed to obtain the needed charge at $10 \mathrm{GeV}$. This accumulation can be done in storage rings because an ion lifetime in these devices, limited mainly by intrabeam scattering, is typically 


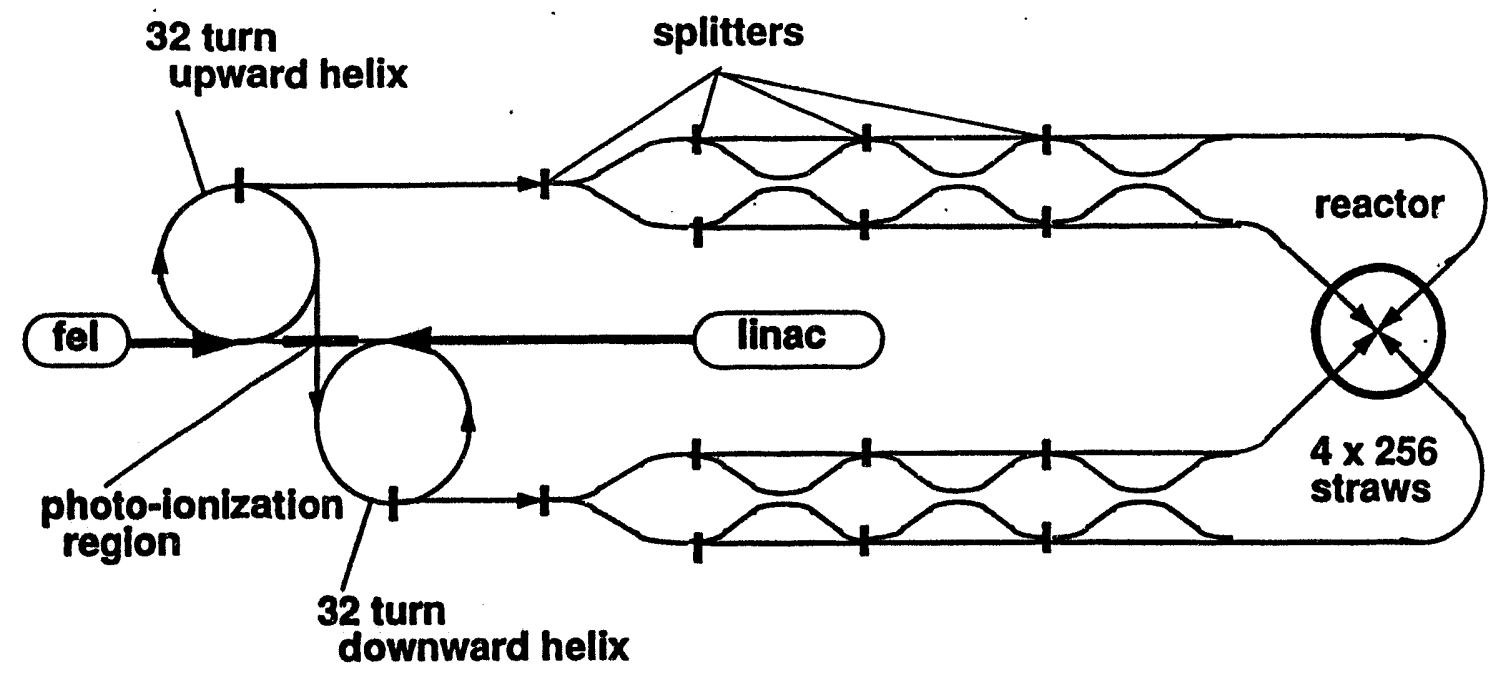

$1-3 \mathrm{~km}$

Fig. 4 Schematic layout of an of driver

around $30 \mathrm{~ms}$. However, since the total beam length is at least $4 \times 10^{5} \mathrm{~m}$, the beams in any sensible system must be stacked. A recent proposal, ${ }^{(4)}$ sketched in Fig. 4, achieves the required time compression in three steps. The current source is a conventional linear if accelerator, which accelerates $50 \mathrm{~mA}$ to the required $10 \mathrm{GeV}$. With a typical acceleration gradient, the accelerating structure or "latticen would be about 1-3 $\mathrm{km}$ in length. The beam is stored for $10 \mathrm{~ms}$ and stacked in a double-helix storage ring, consisting of a 32 loop ascending helix, a 32 loop descending counter-rotating helix, and straight connecting sections at the top and bottom. The beams are stacked in this storage ring using a novel "non-Liouvillian" method, which is discussed subsequently. It is expected that the beam phase-space density in the storage ring can be increased by a factor of about 1000 by this technique with perhaps a $20 \%$ beam loss, leaving an average current of about $40 \mathrm{~A}$ in the ring. The second step in time compression is the simultaneous extraction of the accumulated beam from all 64 loops of the storage ring, giving 64 beams that are about $1.6 \times 10^{-7} \mathrm{~s}$ in duration and $16 \mathrm{~m}$ in length. The final time compression comes from passing each beam through four sets of electrostatic splitters. A splitter consists of a pair of deflecting plates on opposite sides of the incoming beam pipe and a pair of outgoing pipes meeting at a septum. For the first half of the pulse, deflecting plates would drive the beam into one of two outgoing beam pipes, and at the halfway point, the voltage on the plates would reverse, sending the beam to the second pipe. The second beam pipe would have a shorter path length, so the back half of the pulse would catch up with the front before they reach the next set of splitters. After four successive splittings, there would be 1024 beam "straws", each 10 ns in duration and $1 \mathrm{~m}$ long. These straws would finally be focused through four superconducting quadrupole arrays onto four absorbers on the target. Other conceptual designs of rf drivers have been offered, ${ }^{(5,6)}$ but these designs rely similarly on non-Liouvillian beam stacking and differ mainly in details.

\subsection{Beam Stacking}

The principal obstacle to stacking beams in a storage ring is the fact, stated in Liouville's theorem, that the phase-space volume of collisionless particles is conserved, so that the phase-space density of a beam cannot normally be increased by beam merging. Merging beams using conventional focusing elements invariably increases either the spatial or velocity spread of the combined beam and hence the emittance. The alternative stacking method recommended in Ref. 3 is to accelerate singly-charged ions but tune the storage ring for an ion charge $q=+2$. The accelerator beam would overlap one of the straight connecting lines between the storage-ring stacks and would be photo-ionized to $q=+2$ by a counter-propagating ultraviolet laser beam. The doubly-ionized particles are transferred into the storage ring without a change of velocity and can be stacked with previously photo-ionized particles if they are timed to overlap. This process can be particularly efficient with ions, such as $\mathrm{Bi}^{+}$and $\mathrm{Ba}^{+}$, that exhibit sharp resonant peaks in their ionization 
cross-sections.

Photo-ionization has not yet been tested for beam stacking because of the high laser intensity that is needed even for resonant ionization. If $90 \%$ of a singly-charged Barium beam is to be ionized on a single pass through the overlap region with reasonable choices for the beam cross-section and the interaction length, then a laser power of $30 \mathrm{~kW}$ at a wavelength of $840 \mathrm{~nm}$ is required. Continuous power at this wavelength is far beyond the current capabilities of conventional lasers but might be achieved by a two-stage free-electron laser in which third-harmonic radiation from the first stage would be amplified in a second stage(7). Other nonLiouvillian processes are being considered for beam stacking, such as electron cooling, molecular dissociation (e.g. $\mathrm{UO}^{+}+\mathrm{nh} \nu \rightarrow \mathrm{U}^{+}+0$ ), and stimulated recombination (e.g. $\mathrm{Bi}^{++}+\mathrm{e}^{-}+\mathrm{h} \nu \rightarrow \mathrm{Bi}^{+}+2 \mathrm{~h} \nu$ ), but investigation of these processes is still in a preliminary state.

\subsection{Other Considerations}

In addition to beam stacking, there are several other practical issues which must be addressed in developing a commercially acceptable if driver. The first of these is the cost of the required if power. The minimum power is the product of the $10 \mathrm{GeV}$ beam energy and the $40 \mathrm{~mA}$ beam current, which is $400 \mathrm{MW}$. If we generously assume an accelerator efficiency of $80 \%$, then $500 \mathrm{MW}$ of if power is needed. The future cost of if power will have to drop significantly below the current value of about $\$ 0.75 / \mathrm{W}$ at $30 \mathrm{MHz}$ for power plants using rf drivers to provide electricity at a competitive price. Another cost consideration is the number of focusing and bending elements needed to handle up to 1024 beam straws approaching the final focus quadrupoles. Unless the same magnets can be used to manipulate many straws, this final lattice section will be very complicated and costly, due the the large bend fields that are required. (At $10 \mathrm{GeV}$, a field of about $1 \mathrm{~T}$ over a $1 \mathrm{~m}$ path length is needed to bend 200 amu ions by just $1^{\circ}$.) The number of beams straws also raises the question of radiation escape from the reactor chamber along the beam paths. Although less than $1 \%$ of the solid angle around the target is subtended by beam straws, the neutron flux along the beam paths would be sizable. Finally, there are several physics questions that require more theoretical and experimental work, such as the stability of beams in the storage rings, the space-charge repulsion between beams merging in the photo-ionization region, and the effect of debris produced at the splitter septa.

\section{INDUCTION DRIVERS}

\subsection{Induction Accelerators}

An induction accelerator, in effect, is a series of single-turn transformers with the beam serving in place of the secondary windings. The components of an induction cell are shown schematically in Fig. $3 \mathrm{~b}$. In each cell, voltage from a high-power pulse modulator builds up an azimuthal magnetic field $B_{\theta}$ in a ferromagnetic ring or "core," and the changing flux through the core induces an axial electric field $E_{z}$. If the pulsed power to the induction cells is timed appropriately, the $E_{z}$ pulse in each cell coincides with the beam arrival, and the beam is accelerated.

Each ferromagnetic core serves as inductive isolation across the pulse modulator, slowing the increase of $B_{\theta}$ and allowing a longer $E_{z}$ pulse across the acceleration gap. The current through a core remains small until the core becomes saturated. After saturation, the permeability approaches the vacuum value $\mu_{0}$, and the core becomes a low inductance load. For a given voltage $V_{g}$ across a gap, the duration $t_{g}$ of the accelerating pulse is found, from Faraday's equation, to be given by

$$
V_{g} t_{g}=\Delta B_{\theta} A_{c}
$$

where $\Delta B_{\theta}$ is the field change in the core and $A_{c}$ is the cross-sectional area of the core. Eq. (2) shows the advantage of using a ferromagnetic material like METGLAS, ${ }^{(8)}$ with a maximum flux swing of about $2.9 \mathrm{~T}$, instead of ferrite, which typically has a usable flux swing of $0.3 \mathrm{~T}$.

Induction accelerators have several features which make them attractive HIF drivers. The structure has very low impedance, so that currents as high as $100 \mathrm{kA}$ can be accelerated. Furthermore, particles in the gap are accelerated independent of their velocity, and as a result, beams with a head-to-tail velocity 
variation or "velocity tilt" can be accelerated, allowing the beams to compress during acceleration. These features eliminate the need for beam accumulation and splitting found with rf accelerators and lead to relatively simple driver layouts. Since beam manipulations almost invariably increase the 6-D phase space volume, the simpler designs possible with induction accelerators should ease the problem of obtaining a small spot size on targets: Other useful features are a high repetition rate and the fact that several beams can be accelerated through a single ferromagnetic core, reducing the total core cost. A final desirable trait of induction accelerators is that most critical physics issues can be studied using reduced-scale experiments.

The high current possible in induction accelerators makes space charge more important in the transverse dynamics than the emittance. Such beams are said to be "space-charge dominated," and because the spacecharge force counters the external focusing, the net focusing from quadrupoles is substantially reduced and varies along the beam. Another consequence of the high space charge is the interaction of the beam with its image charge in the beam-pipe walls. The resistance of accelerating cells retards the movement of image charges and can cause the growth of electrostatic perturbations on the beam. However, the growth of this "longitudinal instability" has been found to be substantially reduced by the cell capacitance, ${ }^{(9)}$ and the small residual growth should be controllable using "feed-forward" techniques. Magnetic interaction of the beam with its image current is unimportant due to the low beam velocity, and preliminary calculations indicate that beam-beam interactions in accelerating cells should also be negligible.

Unlike if accelerators, the acceleration field in induction cells provides no longitudinal focusing, and without confinement of the ends, space-charge dominated beams would lengthen unacceptably during acceleration. The method usually chosen to confine the beam ends is to impose a suitable time-dependent $E_{z}$

field that reduces acceleration of the head and increases the tail acceleration to just balance the effects of longitudinal spaco-charge. The time-dependent fields at the beam head and tail, referred to as "ears," are proportional to the axial derivative of the beam charge density and to the ratio of the focusing period to the gap length. ${ }^{(10)}$

In addition, the possibility of accelerating beams with a velocity tilt complicates beam manipulations. Since the force applied by bend magnets depends on the beam velocity, beam steering becomes more difficult when a velocity tilt is imposed for compression. Final focus beam optics, in particular, must be designed to be approximately achromatic.

\subsection{Linear Drivers}

A linear layout, sketched in Fig. 5, is commonly proposed for an induction driver ${ }^{(2,11)}$. In this configuration, between 16 and 64 "beamlets" are injected at $3 \mathrm{MeV}$ and are accelerated up to about $50 \mathrm{MeV}$ using electrostatic quadrupole focusing. In this accelerator section, the beam length is compressed from 35 $\mathrm{m}$ to $28 \mathrm{~m}$, corresponding to a decrease of duration from $20 \mu \mathrm{s}$ to $4 \mu \mathrm{s}$ and a current increase from $50 \mathrm{~A}$ to $250 \mathrm{~A}$ per beamlet. The number of beams is chosen so the current in each beam stays below the Maschke limit, given by Eq. (1), for reasonable focusing voltages. An alternative strategy would be to use fewer but longer beams at the same current, but a longer and costlier accelerator section at reduced gradient would then be needed to obtain the same final beam energy and length. A number between 16 and 64 is expected to minimize the driver cost, but a careful study is needed to choose the optimum number. According to Eq. (1), a smaller number of beams is allowed at higher energy and is economically preferable because it reduces the aperture and hence cost of accelerating cores. Consequently, the beamlets are combined in a 4:1 combiner consisting of a series of bends and quadrupoles that orient and bring together the four beamlets. This merging invariably entrains some empty phase space, resulting in additional transverse emittance growth. Computer simulations ${ }^{(12)}$ suggest that the additional transverse emittance increase above the minimum, given by the sum of initial emittances, is less than a factor of four. After merging, the 4-16 beams are then accelerated to $10 \mathrm{GeV}$ using superconducting inagnetic quadrupoles for focusing, while the duration of each pulse is compressed to about $100 \mathrm{~ns}$. Along this acceleration section, which is between $4.5 \mathrm{~km}$ and $9.5 \mathrm{~km}$ long, the acceleration gradient is gradually increased and the focusing period is lengthened to maintain a nearly constant impulse from the quadrupoles as the beam velocity increases. The final compression to a 10 ns pulse duration is achieved by imposing an appropriate velocity tilt and allowing the beam to compress as it drifts along the focusing channel to the reactor chamber. The velocity tilt in this "drift-compression" 


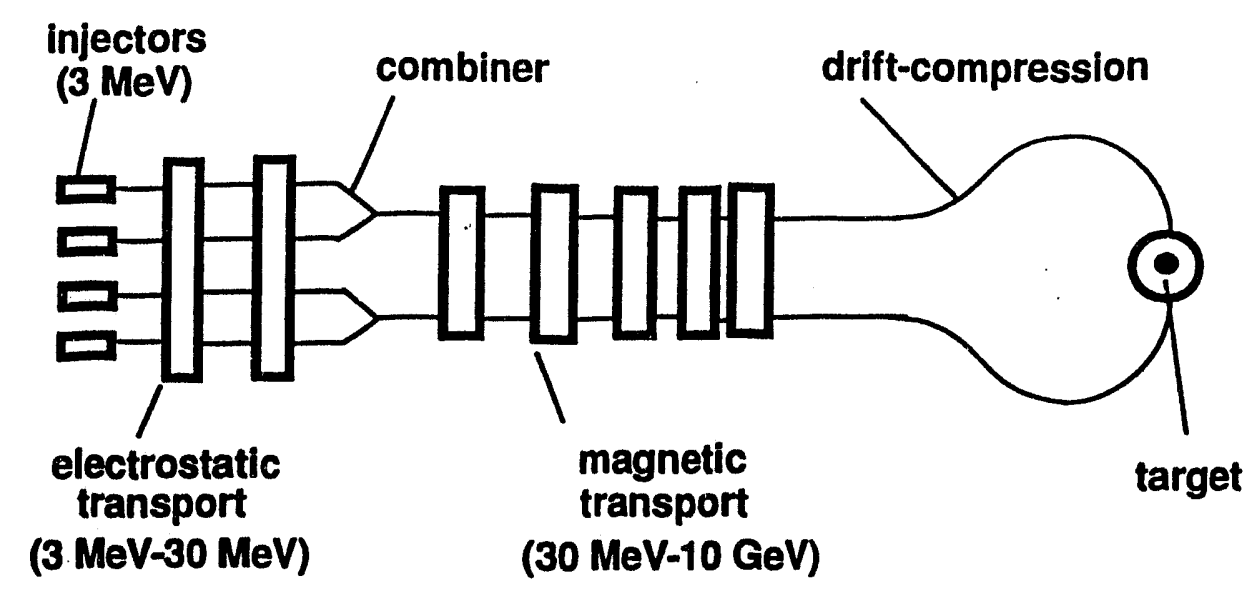

$5-10 \mathrm{~km}$

Fig. 5 Schematic layout of a linear induction driver

section is chosen so that the beam velocity is essentially constant along each pulse when the beams reach the final-focus magnets.

A clear advantage of the linear induction driver compared to proposed $\mathrm{rf}$ drivers is its simplicity. The timing of accelerating cells is determined by the beam time of flight, and aside from the early beam merging, some possible steering and shaping, and the final bending of beam lines to the reactor chamber, there is no beam manipulation. Since beams pass once through each lattice element, the acceleration gradient and the focusing can be optimized at each point, and any debris resulting from beam particles hitting the beam pipe will not affect the beam, as it would in a storage ring. The major physics uncertainties are the emittance growth caused by merging and the severity of the longitudinal instability, but neither problem is expected now to prove crippling. The cost of a linear driver is likely to be a more significant problem. Although proposed designs have not been optimized, the expected $5-10 \mathrm{~km}$ overall length is certain to keep costs high, due mainly to the cost of the ferromagnetic cores. If the amount of core material is minimized by making the field swing $\Delta B$ in Eq. (2) close to twice the saturation field, then losses in the cores increase, reducing the accelerator efficiency. A higher charge state has also been considered to reduce the overall length, but this choice complicates the final-focus dynamics and chamber propagation.

\subsection{Recirculating Drivers}

The recirculating induction accelerator ${ }^{(13)}$ or "recirculator" has been conceived to preserve many of the desirable features of induction accelerators while reducing the cost of a HIF driver. Recirculators offer the prospect of a substantial cost reduction both by reusing their components up to 100 times, thereby requiring fewer components, and by operating at a lower acceleration gradient, which, according to Eq. (2), reduces the volume and cost of ferromagnetic cores. Since the maximum average acceleration gradient in an induction accelerator is about $1 \mathrm{MV} / \mathrm{m}$, the length of a $10 \mathrm{GeV}$ linear driver is roughly $10 \mathrm{~km} / q$, where $q$ again is the beam charge state. In contrast, the size of a recirculator is determined by the bending radius of $10 \mathrm{GeV}$ ions in an average bend field of about $1 \mathrm{~T}$. The minimum recirculator circumference for $200 \mathrm{amu}$ ions is therefore around $1.3 \mathrm{~km} / \mathrm{q}$. Assuming 100 laps per ring, the accelerating gradient is lower than that of a linear driver by more than a factor of ten, allowing a corresponding reduction in the cross-sectional area and cost of ferromagnetic cores. The necessity for time-varying bending magnets and for core pulsers with a higher repetition rate offset some of the these cost savings but a substantial net cost reduction sems likely.

The layout of a three-ring recirculator design is sketched in Fig. 6. The long path length in a recirculator allows greater compression than a linear design, so longer beams can be used initially, and all sections can be practicably focused using magnetic quadrupoles. The lower current associated with longer beams and the lower cell impedance should also reduce the growth of the longitudinal instability. To obtain a high accelerator 


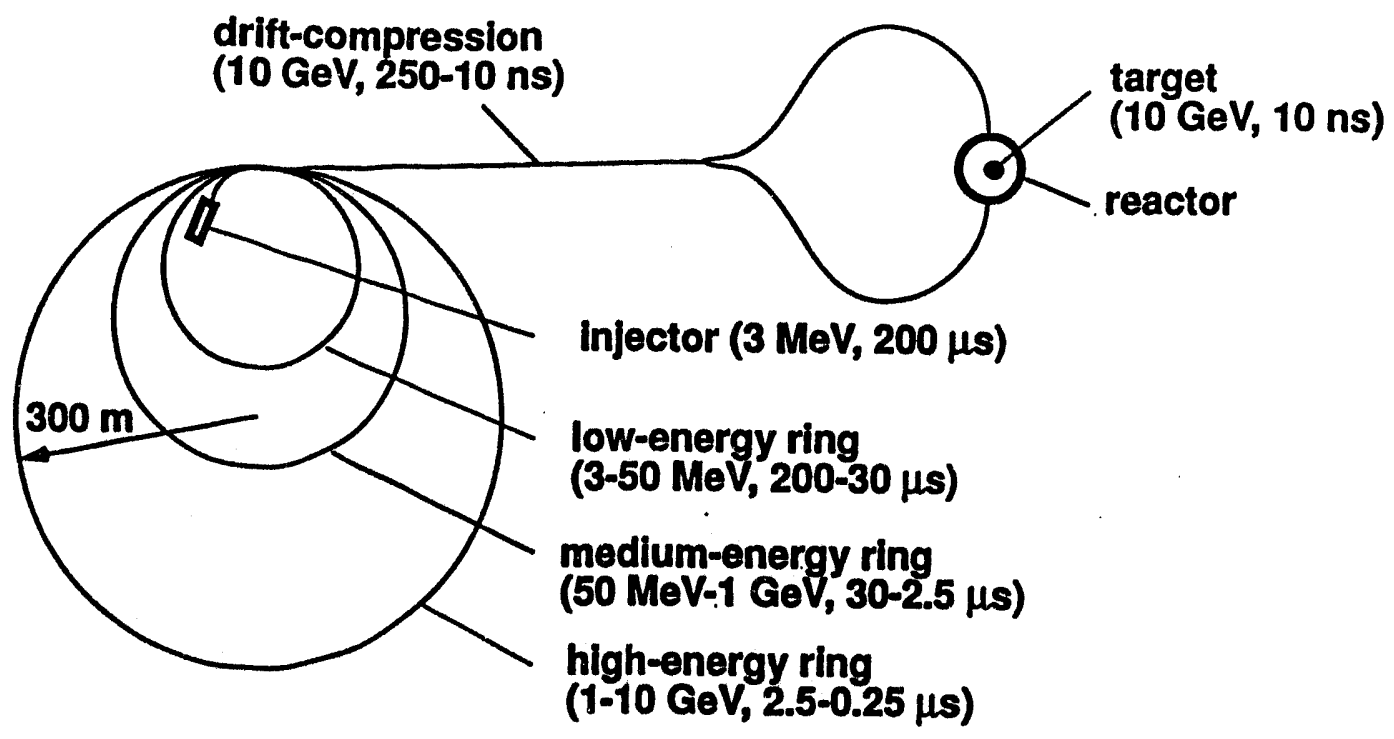

Fig. 6 Schematic layout of a recirculating induction driver

efficiency, these quadrupoles must be superconducting, making the focusing field constant. Multiple rings are then needed because the focusing impulse of magnetic quadrupoles decreases like $V_{b}^{-1}$ due to the decreasing residence time, while the space-charge force increases as the beam is compressed. Together, these changes in the focusing and space charge limit the allowable energy gain in a ring to a factor of at most 20 before the maximum beam radius becomes unacceptably large. For the design in Fig. 6, the energy gain per ring varies between 10 and 20 , and the time compression is a factor between 6.7 and 12 . The drift-compression section in the recirculator is similar to that in a linear driver, except the beam entering it is longer by a factor of 2.5 , making the section somewhat longer. From injection to target, there are four parallel beam lines which pass through the same accelerating cores, but which otherwise have their own focusing and bending magnets. Each ring has a section for inserting the beam into the ring and for extracting it, and between rings there are short matching sections to allow for the changes in lattice period and focusing strength.

While lower cost and the absence of beam merging are important advantages, the recirculator also introduces some complications and physics uncertainties not found in linear drivers. Control and timing of the recirculator bend magnets and accelerating cells are much more challenging. The cell pulsers not only need a much higher repetition rate $(30 \mathrm{kHz}$ vs $10 \mathrm{~Hz})$, but the rate increases in any ring as the beam accelerates. In addition, the ear fields must change from lap to lap'due to compression. The much longer path length in a recirculator and the fact that a beam revisits the same lattice repeatedly dramatically increase the vacuum requirements. Whereas a linear driver is expected to work with a background pressure of $10^{-8}$ Torr, a recirculator will probably need a pressure of $10^{-10}$ Torr to keep beam loss from collisions with the background gas to an acceptable level. The longer path length also requires stricter control of the centroid position. Finally, the recirculator relies on certain beam manipulations that are untested, such as transport of space-charge dominated beams around bends and the insertion of such beams into and extraction from the rings. While theory and simulations are encouraging, experiments are urgently needed to verify that such manipulations are possible.

\section{CONCLUSIONS}

At this time, none of the HIF driver concepts is sufficiently mature that a final choice between them would be warranted. Proposed if drivers use familiar techniques for accelerating and manipulating beams, but the approach appears to be costly, and the proposed method for compression is inefficient and untested. Linear induction drivers offer a much simpler lattice layout and good efficiency, but the merging of highcurrent beams poses a physics uncertainty, and the cost is again an issue. The recirculator promises a 
lower driver cost but entails more complicated controls and greater physics risk. Each concept, moreover, is continuing to develop in response to experiments and numerical modeling.

Reduced-scale experiments to test the three driver concepts are currently underway or are being planned. In Darmstadt, Germany, a heavy-ion synchrotron SIS and an electron-cooled storage ring ESR have been added to an existing of accelerator called UNILAC to test at low current many of the manipulations required in an rf driver. The facility, which became fully operational in 1992, uses highly-ionized heavy ions with energies up to $1 \mathrm{GeV}$ to study such beam dynamics issues as emittance growth, beam stability in rings, intrabeam scattering, and beam merging, although non-Liouvillian stacking and final bunching cannot at present be investigated. Similarly, Lawrence Berkeley Laboratory (LBL) has proposed a $10 \mathrm{MeV}$ test facility, called the Induction Linac Systems Experiments (ILSE), that would examine most of the beam manipulations required in a linear induction driver, including high-current injection, electrostatic and magnetic quadrupole transport, beam nerging, bending, drift-compression, and recirculation. Successful completion of this project would be a major advance for the HIF program. Finally, a proposed series of experiments at Lawrence Livermore National Laboratory in collaboration with LBL seeks to test on a small scale the main physics issues in recirculating induction drivers. Despite the small circumference (about $12 \mathrm{~m}$ ) and the low current (2-8 $\mathrm{mA}$ ), these experiments will test nearly all aspects of recirculator design, including insertion and extraction of space-charge dominated beams in rings, emittance growth during transport in bends, end confinement, steering, and pulse shaping. If these projects are adequately funded, the choice between the three main driver concepts will be much clearer by the end of the decade.

\section{REFERENCES}

1 J. D Lindl, R. McCrory, and E. M. Campbell, Physics Today, 45.9, 32 (Sep 1992).

2 J. Hovingh, V. O. Brady, A. Faltens, D. Keefe, and E. P. Lee, Fusion Technology, 13, 255 (1988).

3 L. W. Alvarez, Phys. Rev., 70, 799 (1946).

4 C. Rubbia, "On Heavy Ions Accelerators for Inertial Confinement Fusion" in Proceedings of the IAEA Technical Committee Meeting on Drivers for Inertial Confinement Fusion, Osaka, Japan, 15-19 April 1991, pp. 23-33 (1992).

5 I. Hofmann, "Key Problems of an Advanced Heavy Ion Fusion Driver," in Proceedings of the IAEA Technical Committee Meeting on Drivers for Inertial Confinement Fusion, Osaka, Japan, 15-19 April 1991, pp. 48-54 (1992).

6 R. W. Müller, "Basic Features of a Reactor Driver Concept for Indirect Drive," in Proceedings of the 3rd European Particle Accelerator Conference, Berlin, Germany, 24-28 March 1992, pp. 1731-1733 (1992).

7 R. Bonifacio, L. De Salvo Souza, P. Pierini, and E. T. Scharlemann, "Generation of XUV Light by Resonant Frequency Tripling in a Two-Wiggler FEL Amplifier," Nucl. Instr. and Meth., A296, 787 (1990).

- 8 METGLAS is a trademark for metal alloys developed by Allied Signal, Inc., Parsippany, NJ.

9 E. P. Lee, Part. Accel. 37-38, 307 (1992).

10 W. M. Sharp, J. J. Barnard, and S. S. Yu, "Pulse Control in an Accelerator for Heavy-Ion Fusion," in Proceedings of the 16th International Linear Accelerator Conference, Ottawa, Canada, 23-28 August 1992, pp. 486-488 (1993).

11 E. P. Lee, "Accelerator and Final Focus Model for an Induction Linac Based HIF System Study" in Proceedings of the 1st International Symposium on Heavy Ion Fusion, Washington, D. C., 26-29 May 1986, pp. 461-473 (1986).

12 C. M. Celata, A. Faltens, D. L. Judd, L. Smith, and M. G. Tiefenback, "Transverse Combining of Nonrelativistic Beams in a Multiple Beam Induction Linac," in Proceedings of the 1987 IEEE Particle Accelerator Conference, Washington, D. C., 16-19 March 1987, pp. 1167-1169 (1987).

13 J. J. Barnard, A. L. Brooks, J. P. Clay, F. E. Coffield, F. J. Deadrick, L. V. Griffith, A. R. Harvey, D. L. Judd, H. C. Kirbie, V. K. Neil, M. A. Newton, A. C. Paul, L. L. Reginato, G. E. Russell, W. M. Sharp, H. D. Shay, J. H. Wilson, and S. S. Yu, "Study of Recirculating Induction Accelerators as Drivers for Heavy Ion Fusion," Lawrence Livermore National Laboratory Report UCRL-LR-108095, May 1992. 


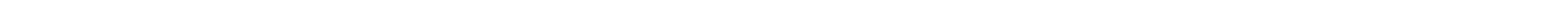



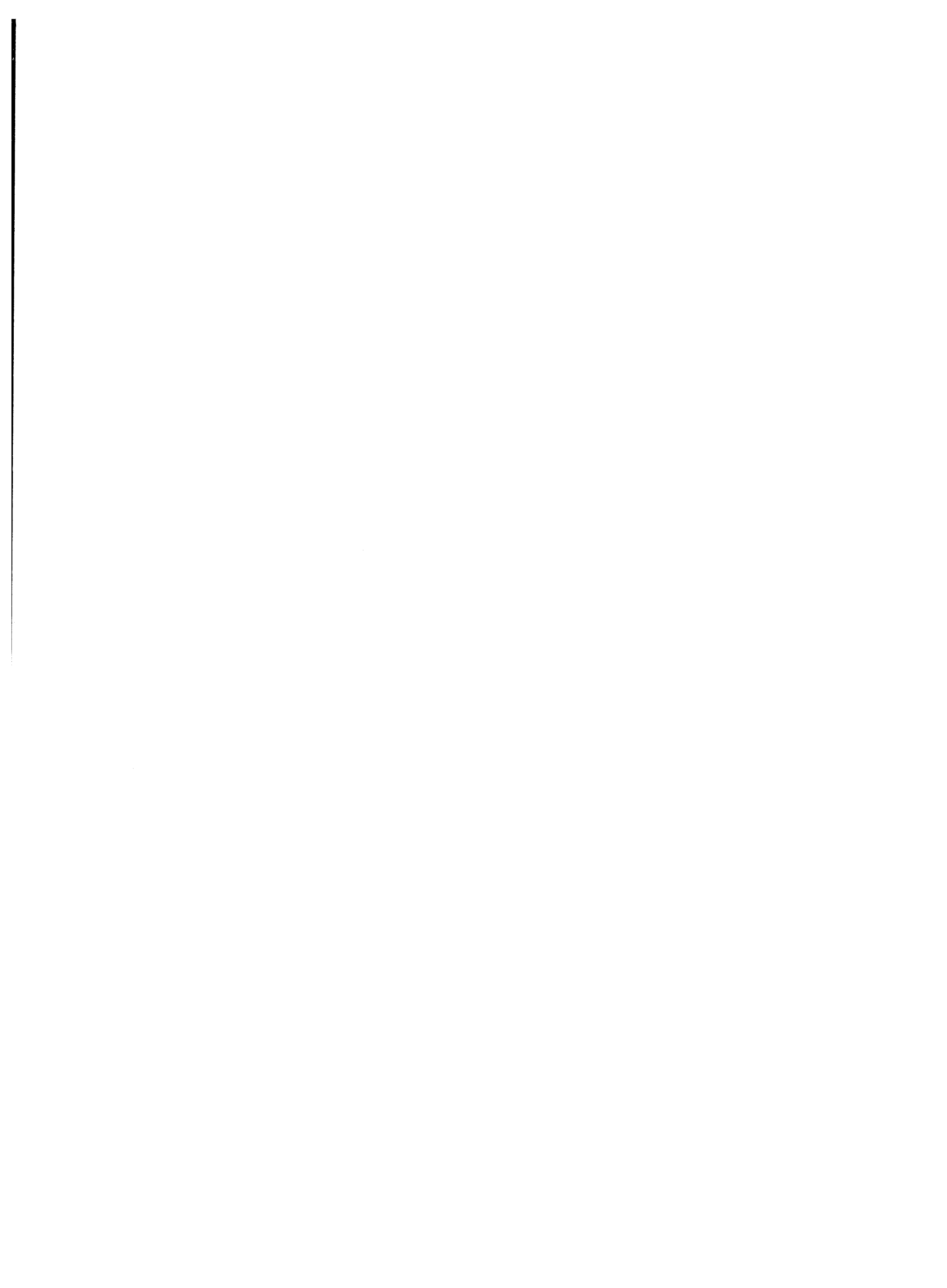

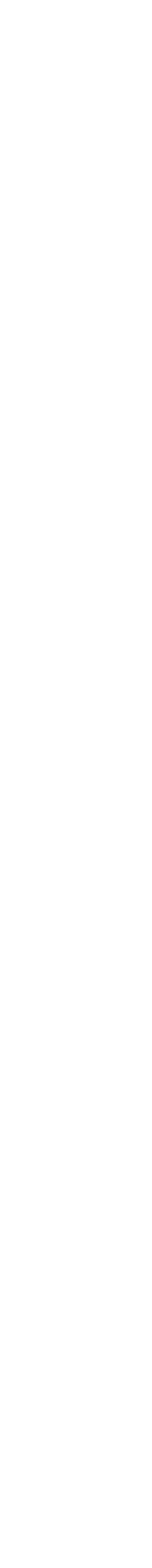
(1)

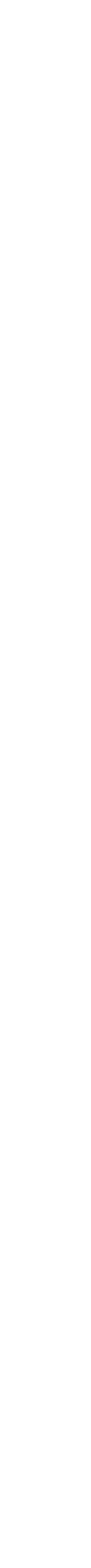

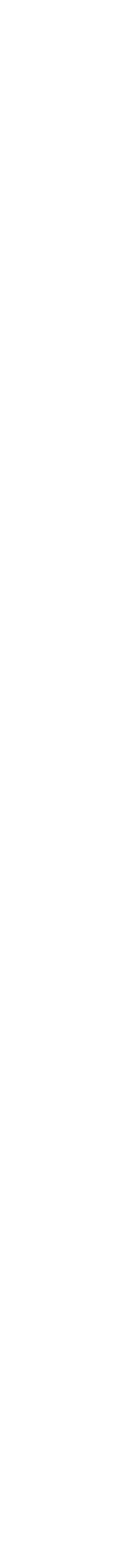

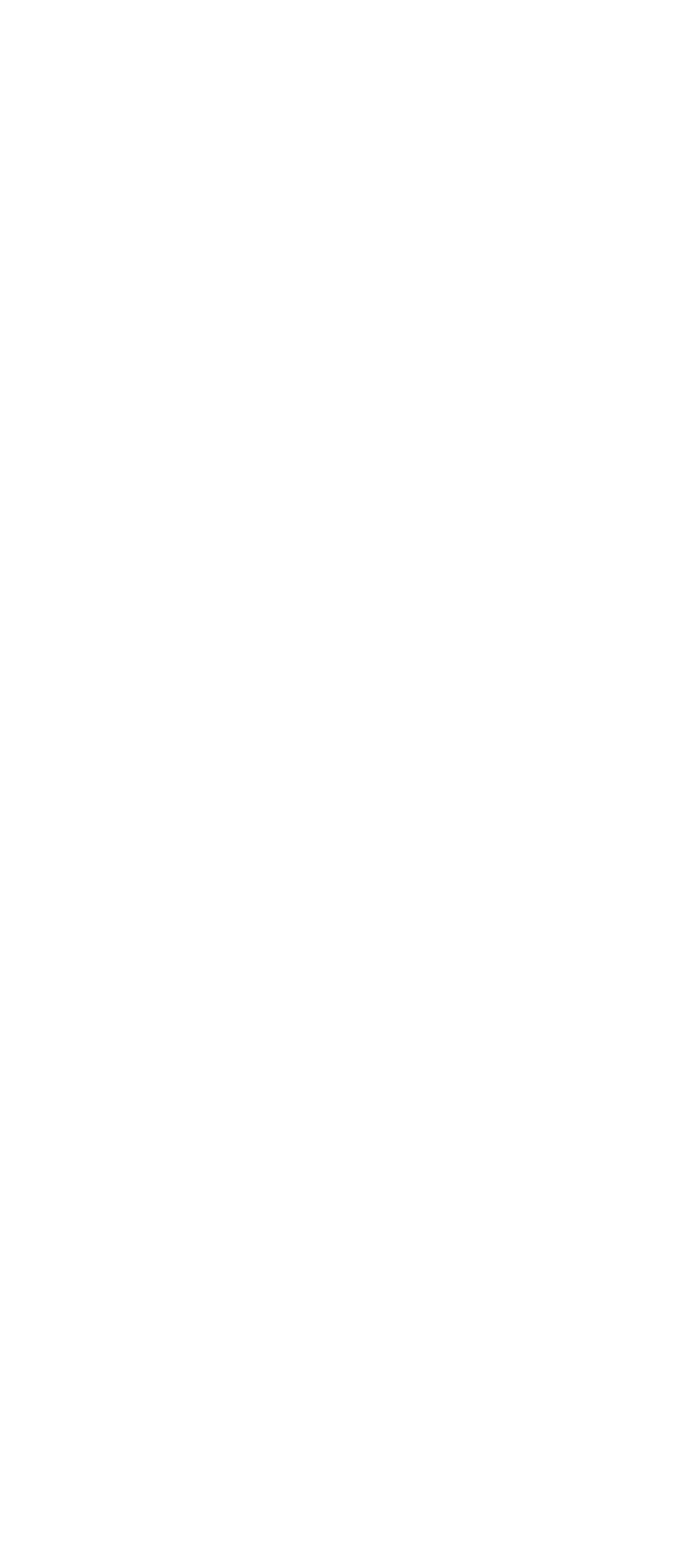

